\title{
MODELO DE FLUJO PARA LA ZONA NO SATURADA: ZONOSA
}

\author{
Gunther Schosinsky \\ Maestría en Manejo de Recursos Hídricos, Escuela Centroamericana de Geología, \\ Universidad de Costa Rica, Apdo. 35, 2060 U.C.R.
}

(Recibido 31/7/1998; Aceptado 28/9/1998)

\begin{abstract}
In Costa Rica, the research of the unsaturated zone began in 1997. The unsaturated zone is important in protecting aquifers from contamination. The understanding of the processes of the unsaturated zone is necessariy to understand the behavior of leakage of landfills or other kinds of surface contamination. For this reason, has been developed a model of the unsaturated zone, which has been called ZONOSA, using an EXCEL worksheet. A model of the unsaturated zone (MARTHE), has been used in a French-Guatemalan study.

The methodology used in the ZONOSA model was the definition of the equations that describes the unsaturated flow in each cell of the worksheet. Each cell represents the defined dimensions of the unsaturated zone. Using very small increments of time, these equations balance the inputs and outputs of water content in each cell.

With an iterative process, the water content, tension and flow for each cell is obtained at a desired time. The model is limited to two layers with of different hydrological properties. The lower layer contains the water table.

A comparison has been made between the results obtained with the MARTHE model and the ZONOSA model. Using the same field data used in the French-Guatemalan study, the ZONOSA model calibrates more accurately to field data of water content at depths of $15 \mathrm{~cm}$ and $130 \mathrm{~cm}$. The MARTHE model, calibrates more accurately to the field data of water content at the depth of $70 \mathrm{~cm}$. This difference is probably due to the fact that the ZONOSA model assumes an isotropic media.

The model is limited to two layers, isotropic media, and each layer is considered homogeneous. Other limitation is that the model works in one dimension and does not consider the horizontal flow in the water content balance.
\end{abstract}

RESUMEN: Las investigaciones en la zona no saturada, se iniciaron en Costa Rica en 1997. La importancia en el estudio de esta zona, es principalmente por ser la que proteje al acuífero de la contaminación. Su conocimiento es fundamental para aclarar el comportamiento de los lixiviados de un basurero u otras fuentes de contaminación sobre el terreno; motivo por el cual, se procedió a la elaboración de un modelo de flujo, que fue denominado ZONOSA, elaborado utilizando el programa de EXCEL. Modelos de la zona no sturada, han sido utilizados en Guatemala por el Proyecto de Cooperación Internacional Franco-Guatemalteco (BRGM-INSIVUMEH, 1997).

La metodología utilizada en el modelo ZONOSA fue definir las ecuaciones en cada celda de la hoja electrónica, representando cada una de ellas con dimensiones definidas de la zona no saturada. Estas ecuaciones contabilizan el balance de entrada y salida de agua en cada celda, en tiempos cortos. Luego con un proceso iterativo se obtiene los cambios de humedad, tensión, caudal etc. para un tiempo deseado. El modelo realiza iteraciones, en períodos de tiempos cortos, calculando el cambio de almacenamiento en cada celda lo que se expresa en la variación del contenido de humedad en cada una de ellas. Es un modelo limitado dos capas con propiedades hidrológicas diferentes cada una de ellas; la capa inferior esta en contacto con el nivel freático.

Comparando el modelo ZONOSA con el MARTHE y utilizando los datos de campo del estudio realizado por el Proyecto de Cooperacion Internacional Franco-Guatemalteco, se deduce que el modelo ZONOSA se ajustó mejor a los datos de campo a las profundidades de $15 \mathrm{~cm}$ y $130 \mathrm{~cm}$. El modelo MARTHE, se ajustó mejor a la profundidad de 70 $\mathrm{cm}$. Esta diferencia se debe posiblemente por considerar el ZONOSA un medio isotrópico. El modelo esta limitado a dos capas y medios isotrópicos, además cada capa es considerada homogénea. Otra limitación se debe a que el modelo es unidimensional, sin considerar el flujo horizontal en el balance de humedades. 


\section{INTRODUCCIÓN}

Las investigaciones en la zona no saturada, se iniciaron en Costa Rica en 1997. La importancia en el estudio de esta zona, es principalmente por ser la que proteje al acuífero de la contaminación. Analizándo la zona no saturada, se puede predecir mediante un modelo el tiempo de llegada de algún contaminante al acuífero. Además, permite conocer los mecanismos de recarga; los cuales se llevan a cabo, a traves de esta zona, la que juega un papel muy importante en la regulación de la misma. Este conocimiento es fundamental para aclarar el comportamiento de los lixiviados de un basurero u otras fuentes de contaminación sobre el terreno, en relación al manto freático y contaminación del acuífero.

Son pocos los modelos conocidos de la zona no saturada existiendo gran necesidad del conocimiento de las leyes que gobiernan su flujo, especialmente para entender el comportamiento de los lixiviados en los basureros, temas de investigación en la Universidad de Costa Rica; motivo por el cual, se procedió a la elaboración de un modelo de flujo para dicha zona, que se ha denominado ZONOSA El modelo es elaborado en una hoja electrónica, utilizando el programa de EXCEL. Una de las ventajas de construir el modelo, ZONOSA, en una hoja electrónica, es que el usuario está generalmente familiarizado con dicho "software" y le permite cambiar algunos parámetro y confeccionar los gráficos deseados.

Modelos similares, han sido utilizados en Guatemala por el Proyecto de Cooperación Internacional Franco-Guatemalteco (BRGM-INSIVUMEH, 1997), auspiciado por la Comunidad Europea, donde la herramienta utilizada para la determinación de las leyes hidrodinámicas, a diferentes profundidades fue el software NONSAT con el modelo MARTHE. Otros autores que han escrito recientemente sobre la zona no saturada son: Candela\& Varela (1993), Fetter (1994) y Frezee \& Cherry (1979). Anteriormente varios autores han escrito sobre el tema, pero es en los últimos veinte años que se ha avanzado enormemente en el conocimiento de dicha zona, impulsado por la necesidad de armonizar el desarrollo humano y la conservarción del recurso de las aguas subterráneas.

La metodología utilizada fue definir las ecuaciones en cada celda de la hoja electrónica, representando cada una de ellas con dimensiones definidas de la zona no saturada. Estas ecuaciones contabilizan el balance de entrada y salida de agua en cada celda, en tiempos cortos. Luego con un proceso iterativo se obtiene los cambios de humedad, tensión, caudal etc. para un tiempo deseado.

\section{MARCO TEÓRICO DE LA ZONA NO SATURADA}

Los suelos cerca de la superficie, rara vez se encuentran saturados, sus poros generalmente tienen agua en forma parcial y el resto es aire. El agua se mantiene en los poros, entre las partículas del suelo, venciendo la gravedad gracias a las fuerzas de tensión superficial. Sin embargo, bajo estas condiciones de suelo no saturado, o parcialmente saturado, se llevan a cabo flujos de agua cumpliendo siempre con la ley de Darcy y la de energía hidráulica de Bernoulli.

En este análisis se ha puesto énfasis en la hidráulica del transporte de agua en la fase líquida. No se discutirá el transporte en la fase de vapor ni la interacción entre suelo, agua y planta; estos tópicos son de interés en el campo agronómico.

Se define el contenido de humedad $\theta=$ $\mathrm{Vw} / \mathrm{Vt}$; o sea, el volúmen de agua contenido en el suelo dividido entre el volumen de suelo. Generalmente se reporta como fración decimal o porcentaje. Para un flujo saturado, el contenido de humedad es igual a la porosidad $(\theta=n)$, mientras que para un flujo no saturado el contenido de humedad es menor que la porosidad $(\theta<n)$

En la zona no saturada el agua que existe en los poros se encuentra a una presión menor de la atmosférica. Generalmente se piensa que el nivel freático es el límite entre la zona saturada y la no saturada. Sin embargo, sobre el nivel freático existe una franja capilar saturada, en que el agua contenida en los poros se encuentra a una presión relativa menor que cero, lo que equivale a una carga de succión, conocida también como tensión. O sea, al igual que en la zona no saturada, 
el agua que se encuentra en la franja capilar presenta presiones menores a la atmosférica; por este motivo la mejor definición del nivel freático es aquella superficie donde la presión del agua contenida en un medio poroso o fracturado es igual a la atmosférica. En este caso, la presión manométrica (p) sería igual a cero. La franja capilar se define como una zona de succión saturada.

De acuerdo a la ecuación de Bernoulli, la carga hidráulica ( $\mathrm{h}$ ) esta dada por:

$\mathrm{h}=\mathrm{p} / \gamma+\mathrm{z}+\mathrm{V}^{2} / 2 \mathrm{~g}$

$\mathrm{h}=$ Carga hidráulica de una partícula de agua expresada en unidades de longitud.

$\mathrm{p} / \gamma=$ Presión equivalente a una columna de agua, de $\mathrm{p} / \psi$ unidades de longitud, que se ejerce sobre una partícula de agua.

$\mathrm{z}=$ Posición de la partícula de agua con respecto a un nivel de referencia arbitrario, expresada en unidades de longitud.

$\mathrm{V}^{2} / 2 \mathrm{~g}=$ Energía cinética de una partícula de agua expresada en unidades de longitud.

Un flujo en medio poroso, al ser laminar, se puede asumir que $\mathrm{V}^{2} / 2 \mathrm{~g}=0$. Si se define $\psi=$ $\mathrm{p} / \gamma$, la carga hidráulica en un medio poroso esta dada por $\mathrm{h}=\psi+\mathrm{z}$. El valor de $\psi>0$ se encuentra por debajo del nivel freático, $\psi=0$ en la superficie del nivel freático y $\psi<0$ en la franja capilar que se encuentra saturada y en la zona parcialmente saturada, conocida como zona no saturada. Para que el agua mantenga una posición en la zona no saturada o en la franja capilar, implica que existen fuerzas de tensión superficial que vencen las fuerzas de gravedad que se ejercen sobre las partículas de agua en el medio poroso. Un análisis microscópico de esta zona revelará meniscos cóncavos que se extienden de grano a grano a través de cada capilar que forman los poros indicando la presencia de cargas de succión o tensión.

La franja capilar se encuentra sobre el nivel estático, en ella los poros están saturados y la presión manométrica es negativa. Por debajo del nivel freático, los poros están saturados y la presión manométrica es positiva.

En la zona por debajo del nivel freático la carga hidráulica $\psi$, debida a la presión, se mide con piezómetros, mientras que en la zona no saturada se mide por medio de tensiómetros, los que consisten en una tasa porosa enterrada bajo la superficie del suelo unida a un tubo, el cual se encuentra lleno de agua y llega hasta la superficie del terreno. En el extremo del tubo que sale sobre la superficie se conecta un vacuómetro. De esta forma el suelo succiona el agua contenida dentro del tubo a traves de la tasa porosa. Al extraer el suelo el agua dentro del tubo, forma un vacío en el extremo superior del mismo, el cual es medido por el vacuómetro. Quedando en equilibrio las fuerzas de vacío y las de succión ejercidas por el suelo.

En la zona no saturada, la conductividad hidráulica K y la carga de succión $\psi$ son función del grado de saturación de la porosidad, aumentando la succión (presión manométrica negativa) cuanto menor sea la saturación del suelo. La permeabilidad aumenta a medida que aumente la humedad en el suelo, siendo la máxima cuando se encuentra saturado.

La ecuación utilizada por BRGM-INSIVUMEH, 1997) para obtener la permeabilidad o la carga de succión es la siguiente:

$\psi=\theta(1 / 2)\left[\left(\theta_{\text {sat }}-\theta\right) /\left(\theta-\theta_{\text {res }}\right)\right]^{\mathrm{c}}$

$\mathrm{K}(\theta)=\mathrm{K}_{\text {sat }}\left[\left(\theta-\theta_{\text {res }}\right) /\left(\theta_{\text {sat }}-\theta_{\text {res }}\right)\right]^{\mathrm{d}}$

$\psi(\theta)=$ Carga de succión para un contenido de agua $\theta$ en la formación. [ L ]

$\psi(1 / 2)=$ Succión a semi saturación

$\theta_{\text {sat }}=$ Contenido de agua a saturación $\left[\mathrm{L}^{3} / \mathrm{L}^{3}\right]$

$\theta_{\text {res }}=$ Contenido de agua residual $\left[\mathrm{L}^{3} / \mathrm{L}^{3}\right]$

$\mathrm{C}=$ Exponente $(0<\mathrm{C}<1)$, relacionado con las características del suelo

$\mathrm{K}(\theta)=$ Permeabilidad para un contenido de agua $\theta$ de la formación $\quad\left[\mathrm{LT}^{-1}\right]$

$\mathrm{K}_{\text {sat }}=$ Permeabilidad a saturación [ LT-1]

$\mathrm{d}=$ Exponente, relacionado con las características del suelo 


\section{MODELO COMPUTARIZADO ZONOSA}

El modelo ZONOSA está elaborado en una hoja electrónica utilizando el programa Excel. Este modelo subdivide el espesor de la capa no saturada hasta el nivel freático en 25 celdas. Posteriormente, utilizando las ecuaciones (2) y (3), el modelo realiza un balance de agua en cada celda, calculando el cambio de almacena- miento de la celda en un tiempo dt; el que es igual al volumen de agua que entra menos el que sale, ambos calculados por Darcy en un tiempo dt. El cambio de almacenamiento afecta el contenido de humedad de la celda en la siguiente forma:

Ejemplo: Cambio de almacenamiento o contenido de humedad, en celda 2 después de un tiempo dt (ecuación 6).

$\mathrm{V}_{2}=(1 / 2)\left[\mathrm{K}\left(\theta_{1}\right)+\mathrm{K}\left(\theta_{2}\right)\right]\left[\mathrm{Z}_{1}+\psi\left(\theta_{1}\right)-\mathrm{Z}_{2}-\psi\left(\theta_{2}\right)\right] \mathrm{Adt}$

$\mathrm{V}_{3}=(1 / 2)\left[\mathrm{K}\left(\theta_{1}\right)+\mathrm{K}\left(\theta_{3}\right)\right]\left[\mathrm{Z}_{3}+\psi\left(\theta_{3}\right)-\mathrm{Z}_{2}-\psi\left(\theta_{2}\right)\right] \mathrm{Adt}$

$\theta_{\mathrm{dt}}=\theta_{2}+\left(\mathrm{V}_{2}-\mathrm{V}_{3}\right) /(100 \mathrm{~L} \mathrm{~A})$

$\mathrm{V}_{2}=$ Volumen de agua que entra en la celda 2 en un tiempo dt

$\mathrm{V}_{3}=$ Volumen de agua que entra en la celda 3 en un tiempo dt

$\theta_{\mathrm{dt}} \quad=$ Humedad en celda 2 después de un tiempo dt

$\theta_{2} \quad=$ Humedad en celda 2

$\mathrm{K}\left(\theta_{1}\right)=$ Permeabilidad de la celda 1 con humedad $\left(\theta_{1}\right)$

$\mathrm{K}\left(\theta_{2}\right) \quad=$ Permeabilidad de la celda 2 con humedad $\left(\theta_{2}\right)$

$\mathrm{K}\left(\theta_{3}\right) \quad=$ Permeabilidad de la celda 3 con humedad $\left(\theta_{3}\right)$

$[\mathrm{L} / \mathrm{T}]$

$\mathrm{Z}_{1} \quad=$ Elevación media de la celda 1 respecto a referencia

$Z_{2}=$ Elevación media de la celda 2 respecto a referencia

$\mathrm{Z}_{3} \quad=$ Elevación media de la celda 3 respecto a referencia

$\psi\left(\theta_{1}\right) \quad=$ Succión en celda 1 con humedad $\left(\theta_{1}\right)$

$\psi\left(\theta_{2}\right)=$ Succión en celda 2 con humedad $\left(\theta_{2}\right)$

$\psi\left(\theta_{3}\right)=$ Succión en celda 3 con humedad $\left(\theta_{3}\right)$

$\mathrm{dt}=$ Tiempo pequeño $\mathrm{dt}$

$\mathrm{L} \quad=$ Espesor de la celda

A $\quad=$ Area de infiltración de la celda

$\left[\mathrm{L}_{2}\right]$

El modelo realiza iteraciones, en períodos de tiempos cortos dt, calculando el cambio de almacenamiento en cada celda lo que se expresa en la variación del contenido de humedad en cada una de ellas.

\section{OPERACIÓN DEL MODELO}

El modelo ZONOSA es un modelo de dos capas con propiedades hidrológicas diferentes cada una de ellas; la capa inferior esta en contac- to con el nivel freático. Al inicio de la hoja electrónica, en las celdas con cursiva, se solicita la información requerida, la que depende de las caracterìsticas físicas de cada capa, tal como se muestra en el cuadro 1.

Antes de ingresar los datos en la hoja electrónica se ha de presionar CRL Q, que acciona la macro para que las operaciones se realicen en modo automático y no se inicien las iteraciones hasta nueva orden. Las celdas debajo del título "VALOR UTILIZADO", son aquellas que pueden ser 
Cuadro 1

Hoja de Excel donde se solicita la información de entrada

\begin{tabular}{|c|c|c|c|c|}
\hline \multirow[b]{2}{*}{ CRL Q Antes de ingresar datos } & \multicolumn{2}{|c|}{ VALOR UTILIZADO } & \multicolumn{2}{|c|}{ VALOR SUGERIDO } \\
\hline & CAPA & CAPA & CAPA & CAPA \\
\hline & SUP. & INF. & SUP. & INF. \\
\hline Humedad de saturación Hs [\% vol] & 30 & 10 & $\mathrm{Hs}<50$ & $\mathrm{Hs}<50$ \\
\hline Cap de campo capa sup. CC [\% vol] & 9,30 & 1,96 & 8,19 & 1,69 \\
\hline Retención específica $\mathrm{Hr}[\%$ vol] & 2 & 0,44 & 2,07 & 0,44 \\
\hline Permeabilidad saturada Ksat [m/seg] & $5,0 \mathrm{E}-06$ & $5 \mathrm{E}-04$ & $\mathrm{~K}>5 \mathrm{E}-8$ & $\mathrm{~K}>5 \mathrm{E}-8$ \\
\hline Succión media $\&(1 / 2)<0[\mathrm{~m}]$ & $-1,8$ & -2 & $-1,79$ & $-1,25$ \\
\hline Coeficiente succión z $0<c<1$ & 0,59 &,- 5 & 0,59 &,- 59 \\
\hline Coeficiente permeabilidad $\mathrm{d} d>0$ & 2 & 2 & & \\
\hline Tipo de formación (tres siglas) & TOB & LAV & & \\
\hline TERMINOS GENERALES & UTILIZADO & & SUGERIDO & \\
\hline Profundidad del nivel freático [m] & 5 & & & \\
\hline Area de infiltración A $\left[\mathrm{m}^{2}\right]$ & 1 & & & \\
\hline Infiltr. - Evap. Pot. INF-ETP [m/seg] & $0,0 \mathrm{E}+00$ & & & \\
\hline Punto marchitez capa sup. PM [\% vol] & 4,6 & & 4,65 & \\
\hline Espesor primer capa ( ? ) [m] & 15 & & & \\
\hline Iniciar con Ho (s/n ?) & $\mathrm{n}$ & & & \\
\hline Iniciar con Dias $=0(\mathrm{~s} / \mathrm{n} ?)$ & $\mathrm{n}$ & & & \\
\hline División última mínima (n/valor ?) & $\mathrm{n}$ & valor $>=$ & 0,010 & \\
\hline Intervalo tiempo (dt iteración) [seg] & 50 & iniciar con & 5,0 & \\
\hline \multirow{2}{*}{\multicolumn{5}{|c|}{$\begin{array}{l}\text { Ingresar datos de humedad con Col. L } \\
\text { Ingresados datos CTRL Z, F9 para correr }\end{array}$}} \\
\hline & & & & \\
\hline Días iterados = 15,87 & & & & \\
\hline
\end{tabular}

modificadas por el usuario, las otras celdas se encuentran protegidas. La retención específica (Hr) se refiere al punto higroscópico, que sería la humedad mínima que puede contener el suelo. La succión media, \&(1/2) es una constante expresada en la ecuación (1) como $\psi(1 / 2)$; este valor, al igual que las constantes "d" y "c" dependen del suelo y se obtienen cuando se calibra el modelo. La recarga (INF-ETP) es la infiltración menos la evapotranspiración potencial (ETP); en otras palabras, es la cantidad de agua que profundiza a las celdas inferiores; si el valor es negativo indica que la evapotranspiración es mayor que la recarga de lluvia. La capacidad de campo (CC), es utilizada por el modelo para proceder a calcular la evapotranspiración proporcional entre CC y el punto de marchitez (PM). De tal forma que el suelo con una humedad igual o mayor a la capacidad de campo, la evapotrans- piración es la potencial. Si el suelo se encuentra a punto de marchitez, o menor, la evapotranspiración es igual a cero.

El modelo solicita la profundidad del nivel freático. Por ser de dos capas, se requiere la profundidad del primer estrato. Si el espesor de este estrato es mayor que el nivel freático el modelo trabaja con una sola capa a una profundidad del nivel freático. Si el nivel freático es mayor que el espesor de la primer capa, el modelo trabaja con dos capas. Hay que considerar que el modelo genera 25 celdas en profundidad, asignándole a cada celda su espesor. A las dos primeras celdas, se le asigna un espesor de la mitad de las otras. A la última celda le asigna un espesor, que define el usuario en la casilla de "Div. última mín.", con el propósito que las celdas restantes sean más delgadas (el espesor asignado será la profundidad al nivel freático menos Div. última dividido entre 
24); de esta forma podrá escoger el espesor de las celdas superiores a la última . Si el usuario desea que el espesor de las celdas hasta la napa freática sea el mismo, asignara una letra " $n$ " en la casilla de "Div. última mín". El usuario ha de ingresar el intérvalo de tiempo a utilizar para cada iteración. El programa recomienda un valor de intérvalo de tiempo a utilizar para iniciar con las iteraciones, sin embargo este valor puede ser cambiado a discreción, posteriormente, para acelerar el programa. Además del tiempo, otros valores son sugerídos; sin embargo pueden variarse para ajustar el modelo.

Para iniciar el modelo, hay que ingresar los valores de la humedad original en cada celda en la columna "Ho" del cuadro 2; si el usuario desea considerarlos en el cálculo del modelo, ha de cambiar la N por una $\mathrm{S}$ y oprimir Enter. Aparecerá un rótulo que dice operación ejecutada, entonces el usuario sustituirá la S por una N. De igual forma se ha de proceder si se desea inicializar el control de los días iterados.

El modo de operación es en forma iterativa y cálculo manual. El primer paso es indicarle al programa el modo de cálculo iterativo, que se ejecuta con una macro presionando control $\mathrm{Z}$ simultáneamente. El número de iteraciones son 30.000. En cada iteración el modelo recorre un tiempo igual al incremento de tiempo asignado. En el ejemplo del cuadro 1 , el tiempo asignado es de 50 segundos; en 100 iteraciones el tiempo recorrido será de 5000 segundos. El cálculo se inicia presionando la tecla F 9. Para cambiar el número de iteraciones en el programa, el menú se encuentra en Herramientas, opciones, calcular. Para detener las iteraciones se presiona la tecla Esc.

El usuario puede cambiar el espesor de las celda (Cuadro 2). Para esto deberá quitar la protección del programa, lo que se hace en el menú Herramientas, proteger, desproteger hoja. El programa tiene varias columnas y filas ocultas, las cuales pueden quedar a la vista, marcando el rango de columnas con el mouse; una vez marcadas, en el menú seleccionar rango, fila o columna según sea el caso y luego mostrar.

En caso de cometer un error en los números de entrada, el programa pondrá una nota al respecto y asignará por su cuenta un valor conveniente. Por ejemplo, si una humedad asignada es mayor que la humedad de saturación, el programa automáticamente asignará un valor entre la humedad de saturación y la humedad de retención específica; además pondrá una nota al respecto.

Si durante el cálculo una celda tiene una succión mayor que la atmosférica, el programa asignará automáticamente una succión de 10 metros, que sería la máxima succión posible en una columna de agua ya que equivale a una atmósfera. Si el programa calcula una humedad menor que la humedad de retención el programa asignará el valor de retención, por no ser aceptables humedades menores a esta. Igualmente sucede si el cálculo de una celda, sobrepasa en humedad al valor de saturación, automáticamente se le asignará a esta celda dicho valor.

El programa contabiliza la recarga al nivel freático, lo que se observa en la última fila del cuadro 2, que muestra un valor de recarga de $0.742 \mathrm{~m}^{3}$, que es el volumen de agua que ha pasado al nivel freático en 16,03 días.

En el cuadro 1, el nivel freático se encuentra a 5 metros de profundidad y el espesor de la primer capa de 15 metros. Por ser menor la profundidad del nivel freático que el espesor de la primer capa, el modelo trabajará en una sola capa.

El programa tiene una hoja GRAF, donde se pueden observar los gráficos de humedad, tensión, caudal ascendente (negativo) o descendente (positivo), potencial hidráulico y permeabilidad a diferentes profundidades. Los resultados se muestran en la figura 1.

En el cuarto gráfico de la figura 1, se muestra el caudal a cada nivel del estrato. Cuando el caudal es negativo, el flujo asciende a los niveles superiores, cuando es positivo el flujo es descendente, como es el caso de la figura 1, en que todas las celdas el caudal es descendente. Estos gráficos se ajustan a sus nuevos valores, una vez que termine el número de iteraciones asignadas. Si se para el proceso iterativo mediante la tecla ESC, los gráficos no se ajustarán automaticamente.

En la Hoja GRAF, al pie de los gráficos se lleva un control de los días iterados, de la recarga al acuífero y de la división de la última celda recomendada, que nos sirve para verificar la convergencia del modelo. 
Cuadro2

Humedad inicial y valores calculados por el programa

\begin{tabular}{|c|c|c|c|c|c|c|c|c|c|}
\hline $\begin{array}{l}\mathrm{Ho} \\
\%\end{array}$ & Capa & $\begin{array}{c}\text { Prof } \\
\mathrm{m}\end{array}$ & $\begin{array}{l}\mathrm{Ho} \\
\%\end{array}$ & $\begin{array}{l}\mathrm{H} \\
\%\end{array}$ & $\begin{array}{c}\mathrm{q} \\
\mathrm{m} / \mathrm{seg}\end{array}$ & $\begin{array}{c}\mathrm{K} \\
\mathrm{m} / \mathrm{seg}\end{array}$ & $\begin{array}{c}\mathrm{q}^{*} \mathrm{~A}^{*} \mathrm{dt} \\
\mathrm{m}^{3}\end{array}$ & $\begin{array}{l}\& \\
\mathrm{~m}\end{array}$ & $\begin{array}{c}\text { Poten. } \\
\text { m }\end{array}$ \\
\hline & TOB & 0,00 & & & & & & & \\
\hline 30 & TOB & $-0,10$ & 30,00 & 6,34 & $0 \mathrm{E}+00$ & $1,2 \mathrm{E}-07$ & $0,0 \mathrm{E}+00$ & $-4,893$ & $-5,0$ \\
\hline 30 & TOB & $-0,20$ & 30,00 & 6,47 & $4 \mathrm{E}-11$ & $1,3 \mathrm{E}-07$ & 2,2E-09 & $-4,793$ & $-5,0$ \\
\hline 30 & TOB & $-\mathrm{O}, 40$ & 30,00 & 6,75 & $9 \mathrm{E}-11$ & $1,4 \mathrm{E}-07$ & $4,5 \mathrm{E}-09$ & $-4,593$ & $-5,0$ \\
\hline 30 & TOB & $-\mathrm{O}, 60$ & 30,00 & 7,06 & $2 \mathrm{E}-10$ & $1,6 \mathrm{E}-07$ & $9,5 \mathrm{E}-09$ & $-4,393$ & $-5,0$ \\
\hline 30 & TOB & $-\mathrm{O}, 8 \mathrm{O}$ & 30,00 & 7,39 & $3 \mathrm{E}-10$ & 1,9E-07 & $1,5 \mathrm{E}-08$ & $-4,194$ & $-5,0$ \\
\hline 30 & TOB & $-1,00$ & 30,00 & 7,76 & $4 \mathrm{E}-10$ & $2,1 \mathrm{E}-07$ & $2,0 \mathrm{E}-08$ & $-3,994$ & $-5,0$ \\
\hline 30 & TOB & $-1,20$ & 30,00 & 8,17 & $5 \mathrm{E}-10$ & $2,4 \mathrm{E}-07$ & 2,6E-08 & $-3,794$ & $-5,0$ \\
\hline 30 & TOB & $-1,40$ & 30,00 & 8,62 & $6 \mathrm{E}-10$ & $2,8 \mathrm{E}-07$ & $3,2 \mathrm{E}-08$ & $-3,595$ & $-5,0$ \\
\hline 30 & TOB & $-1,60$ & 30,00 & 9,12 & $8 \mathrm{E}-10$ & $3,2 \mathrm{E}-07$ & $3,8 \mathrm{E}-08$ & $-3,395$ & $-5,0$ \\
\hline 30 & TOB & $-1,80$ & 30,00 & 9,68 & $9 \mathrm{E}-10$ & 3,8E-07 & 4,4E-08 & $-3,196$ & $-5,0$ \\
\hline 30 & TOB & $-2,00$ & 30,00 & 10,30 & $1 \mathrm{E}-09$ & 4,4E-07 & 4,9E-08 & $-2,996$ & $-5,0$ \\
\hline 30 & TOB & $-2,20$ & 30,00 & 11,00 & 1E-09 & $5,2 \mathrm{E}-07$ & $5,5 \mathrm{E}-08$ & $-2,797$ & $-5,0$ \\
\hline 30 & TOB & $-2,40$ & 30,00 & 11,78 & 1E-09 & $6,1 \mathrm{E}-07$ & $6,1 \mathrm{E}-08$ & $-2,597$ & $-5,0$ \\
\hline 30 & TOB & $-2,60$ & 30,00 & 12,66 & $1 \mathrm{E}-09$ & $7,3 \mathrm{E}-07$ & $6,6 \mathrm{E}-08$ & 2.398 & $-5,0$ \\
\hline 30 & TOB & $-2,80$ & 30,00 & 13,65 & $1 \mathrm{E}-09$ & $8,7 \mathrm{E}-07$ & $7,2 \mathrm{E}-08$ & $-2,198$ & $-5,0$ \\
\hline 30 & TOB & $-3,00$ & 30,00 & 14,76 & $2 \mathrm{E}-09$ & 1E-06 & 7,7E-08 & $-1,998$ & $-5,0$ \\
\hline 30 & TOB & $-3,20$ & 30,00 & 16,01 & 2E-09 & $1,3 \mathrm{E}-06$ & $8,1 \mathrm{E}-08$ & $-1,799$ & $-5,0$ \\
\hline 30 & TOB & $-3,40$ & 30,00 & 17,40 & 2E-09 & $1,5 \mathrm{E}-06$ & $8,5 \mathrm{E}-08$ & $-1,599$ & $-5,0$ \\
\hline 30 & TOB & $-3,60$ & 30,00 & 18,94 & 2E-09 & $1,8 \mathrm{E}-06$ & 8,9E-08 & $-1,399$ & $-5,0$ \\
\hline 30 & TOB & $-3,80$ & 30,00 & 20,64 & 2E-09 & 2,2E-06 & $9,3 \mathrm{E}-08$ & $-1,199$ & $-5,0$ \\
\hline 30 & TOB & $-4,00$ & 30,00 & 22,45 & 2E-09 & 2,7E-06 & $9,6 \mathrm{E}-08$ & $-0,999$ & $-5,0$ \\
\hline 30 & TOB & $-4,20$ & 30,00 & 24,35 & 2E-09 & $3,2 \mathrm{E}-06$ & $9,8 \mathrm{E}-08$ & $-0,8$ & $-5,0$ \\
\hline 30 & TOB & $-4,40$ & 30,00 & 26,24 & 2E-09 & $3,7 \mathrm{E}-06$ & $1,0 \mathrm{E}-07$ & $-0,6$ & $-5,0$ \\
\hline 30 & TOB & $-4,60$ & 30,00 & 27,97 & 2E-09 & $4,3 \mathrm{E}-06$ & $1,0 \mathrm{E}-07$ & $-0,4$ & $-5,0$ \\
\hline 30 & TOB & $-4,80$ & 30,00 & 29,34 & 2E-09 & $4,8 \mathrm{E}-06$ & $1,0 \mathrm{E}-07$ & $-0,2$ & $-5,0$ \\
\hline 30 & N.E. & $-5,00$ & 30,00 & 30,00 & 2E-09 & 5E-06 & $1,0 \mathrm{E}-04$ & 0 & $-5,0$ \\
\hline 30 & TOB & $-5,20$ & 30,00 & 30,00 & & $5 \mathrm{E}-06$ & $2,5 \mathrm{E}-04$ & $0 \&$ & $-5,2$ \\
\hline Ho & & & & & & & & & \\
\hline & & Prof & $\mathrm{HO}$ & $\mathrm{H}$ & $\mathrm{q}$ & K & $\mathrm{q}^{*} \mathrm{~A} * \mathrm{dt}$ & $\&$ & Poten. \\
\hline Ho & & $\mathrm{m}$ & $\%$ & $\%$ & $\mathrm{~m} / \mathrm{seg}$ & $\mathrm{m} / \mathrm{seg}$ & $\mathrm{m}^{3}$ & $\mathrm{~m}$ & $\mathrm{~m}$ \\
\hline & & \multicolumn{8}{|c|}{$\begin{array}{l}\text { RECARGA } 0,742 \mathrm{~m}^{3} \\
\text { TIEMPO } 16,03 \text { días }\end{array}$} \\
\hline
\end{tabular}

$\begin{array}{ll}\mathrm{H}_{0} & =\text { Humedad original medida en el campo \% } \\ \mathrm{H} & =\text { Humedad calculada } \% \\ \mathrm{q} & =\text { Caudal específico Q/A m } 3 / \mathrm{seg} / \mathrm{m}^{2} \\ \text { vol. entra } & =\text { Volumen que entra a la celda } \\ \& & =\text { Succión en metros } \\ \text { Poten. } & =\text { Potencial }(\mathrm{Z}+\&) \text { en } \mathrm{m} \\ \mathrm{PM} & =\text { Punto de Marchitez } \% \\ \mathrm{INF} & =\text { Infiltración } \mathrm{m} / \mathrm{seg} \\ \mathrm{K} & =\text { Permeabilidad } \mathrm{m} / \mathrm{seg} \\ \mathrm{A} & =\text { Area de infiltración, generalmente } 1 \mathrm{~m}^{2} \\ \mathrm{dt} & =\text { Tiempo escogido para cada iteración seg }\end{array}$




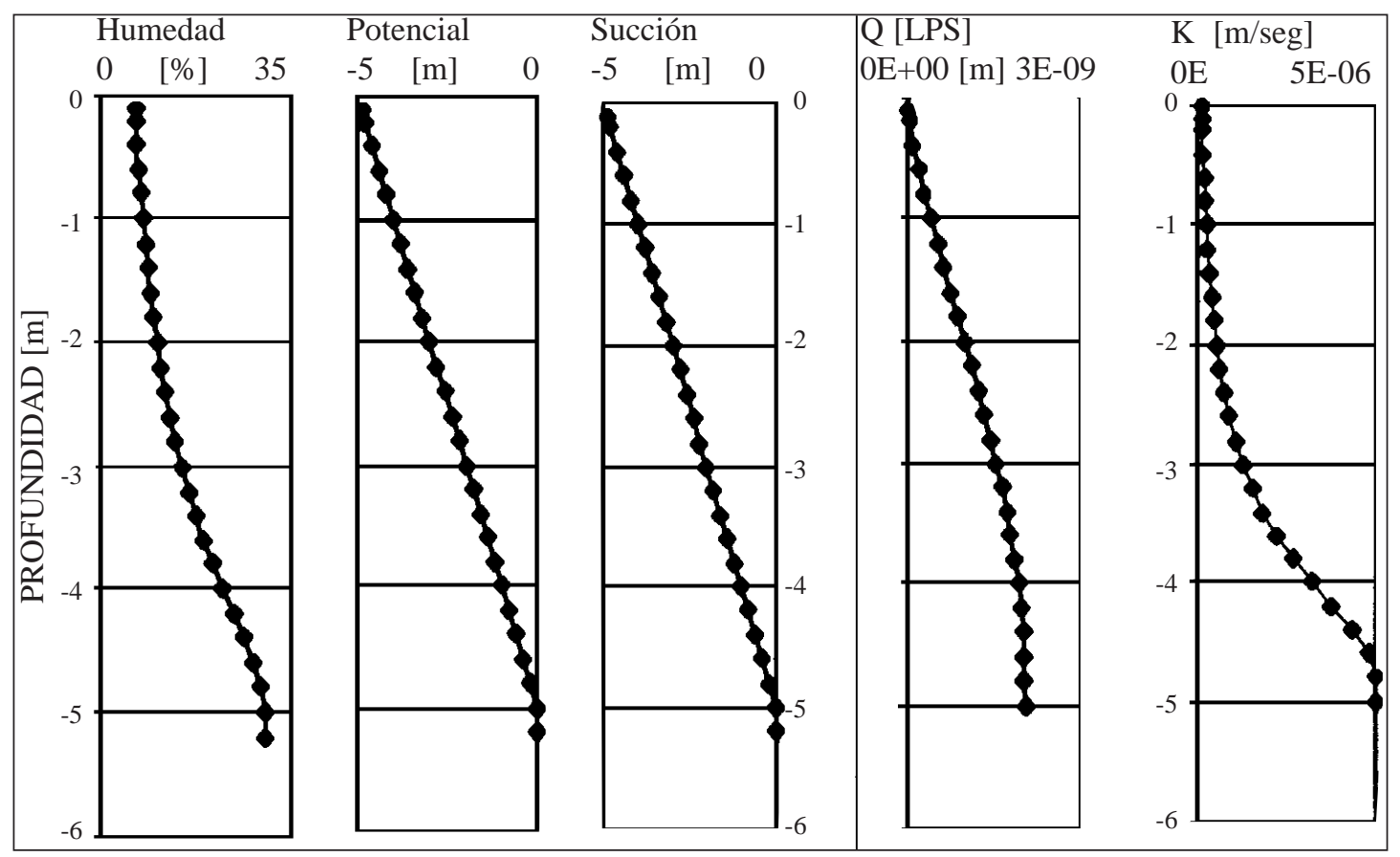

Fig. 1: Gráficos de humedad, succión, potencial, caudal y permeabilidad a diferentes profundidades. Nivel freático a 5 metros de profundidad. Estos gráficos son el resultado de los valores ingresados en el Cuadro 1 y mostrados en el Cuadro 2. Tiempo = 16,03 días, recarga = 0,742 $\mathrm{m} 3$, división última $=0,01$.

\section{Convergencia del modelo}

Uno de los factores que más influye en la convergencia, son los valores de la humedad original de la zona no saturada. Estos valores pueden ser no posibles de acuerdo a los datos de entrada, produciendo problemas de convergencia. Otro factor que influye es el incremento de tiempo seleccionado. Valores de incremento de tiempo altos con respecto a los datos de entrada, producirán problemas de convergencia. Se recomienda iniciar con incrementos de tiempo bajo, utilizando el valor recomendado. Posteriormente este incremento de tiempo se puede ir aumentado para aligerar el proceso. La no convergencia se nota por los cambios constantes, en forma ascendente y descendente de los datos de humedad, tensión, caudal y permeabilidad en una o varias celdas. En una buena convergencia la tendencia ascendente o descendente de los valores en las celdas, se mantiene hasta llegar a estabilizarse con el tiempo.

Un valor que mejor muestra la convergencia del modelo es la división última recomenda- da. Si esta división última esta variando constantemente de valor, en forma ascendente y descendente, indica la no convergencia del modelo. Algunas recomendaciones para converger al modelo son las siguientes:

1- Si no se conocen los datos de humedad original de la zona no saturada, asumir una porosidad saturada originalmente y correr el modelo con el incremento de tiempo recomendado. Cuando los valores se encuentran más o menos estabilizados, proceder a aumentar el incremento de tiempo lentamente. En caso de que no se produzca la convergencia disminuir el incremento del tiempo.

2- Después de haber corrido el modelo, y se encuentre más o menos estable, no más de dos meses (época seca), copiar los datos de humedad obtenidos por el modelo, como los valores de la humedad original, sabiendo que son los que corresponden al final de la época seca. 
3- Introducir la recarga de lluvia correspondiente a los datos de evapotranspiración inicializar los tiempos y las humedades originales. Luego, correr el modelo con el incremento de tiempo recomendado inicialmente, para ir aumentando poco a poco el incremento del tiempo. Recuerde que uno de los indicadores de la convergencia, es que la división última recomendada se estabilice.

4- Para acelerar el modelo, ubicarse en la hoja GRAF, y desde allí correr el modelo, lo que producirá iteraciones más rápidas. En esta hoja se tiene la información del tiempo iterado, la recarga al acuífero y división última, para observar la convergencia.

\section{Calibración del Modelo}

Para calibrar el modelo, han de ingresarse correctamente los valores solicitados por el modelo, los que se encuentran en el cuadro 1 y 2 en las columnas "Valor utilizado" y "Ho", respectivamente.

Los valores recomendados en el cuadro 1 , están basados en la humedad y permeabilidad saturada. Con estos valores, el programa recomienda los valores de capacidad de campo, intérvalo de tiempo, valor de la tensión media, punto de marchitez y humedad de retención. Cualquiera de estos valores pueden ser cambiados si el usuario lo considera necesario. Un cambio en la capacidad de campo afectará al resto de los valores recomendados. El valor recomendado de capacidad de campo, utilizando el valor de la humedad de saturación ingresada por el usuario. Los valores recomendados de humedad de retención y punto de marchitez se calcularon en base a la capacidad de campo $(\mathrm{PM}=\mathrm{CC} / 2 ; \mathrm{Hr}=\mathrm{CC} / 4,5)$. Sabiendo que la capacidad de campo corresponde a una succión de 1/3 de atmósfera y el punto de marchitéz a 15 atmósferas, se obtuvo un valor del coeficiente de succión $\mathrm{c}=0,59$ para todos los suelos y el valor de la succión media dependiente de la humedad de saturación y capacidad de campo.

Con los siguientes datos de campo: humedad original del suelo, infiltración que ingresa por lluvia, evapotranspiración potencial, hume- dad y tensión del suelo a diferentes tiempos y profundidades; se puede calibrar el modelo, de tal forma que reproduzca los datos de campo medidos, variando los valores solicitados en el cuadro 1.

Los cambios de valores en el cuadro 1 producirán los siguientes efectos:

\section{1- Succión media [\&1/2]}

A mayor valor absoluto de $\& 1 / 2$ la succión en las celdas es mayor, aumentando casi proporcionalmente, entre rangos negativos menores a 10, que sería el valor mayor a utilizar. Un valor general utilizado en tobas arcillosas es $\& 1 / 2=-4$ mts. Para aluviones utilizar valores menores y para arcillas mayores.

\section{2- Coeficiente de Succión [c]}

A menor coeficiente de succión "c", la succión en las celdas se uniformiza aproximándose a la succión media $(\& 1 / 2)$, aún existiendo en las celdas variaciones de humedad. Un valor a utilizar en tobas es de $\mathrm{c}=0,15$. En aluviones utilizar valores menores y en arcillas mayores.

A mayor coeficiente de succión, la succión forma rangos de varios órdenes de magnitud, disminuyendo la succión donde la humedad se acerca a la saturada; o sea, por debajo de la humedad media, y aumentando la succión donde la humedad es menor de la media.

\section{3- Coeficiente de permeabilidad [d]}

A menor coeficiente de permeabilidad “d”, la permeabilidad en las celdas se uniformiza aproximándose a la permeabilidad saturada aún habiendo grandes variaciones de humedad. Un valor a utilizar en tobas es $d=4$. En aluviones utilizar valores menores y en arcillas mayores.

A mayor coeficiente de permeabilidad, esta forma rangos con valores cerca de la permabilidad saturada en las celdas donde la humedad es mayor y más baja donde la humedad es menor, disminuyéndose considerablemente.

Para fines de orientación, con el propósito de que el usuario pueda alimentar con valores típicos el modelo, se presentan en el cuadro 3 algunos valores de permeabilidad saturada, valores de capacidad de campo y punto de marchitez. 
Cuadro 3

Valores de capacidad de permeabilidad, capacidad de campo y punto de machitez, de algunos suelos.

(Tomado de Jegat, com. esc., 1979)

\begin{tabular}{lcccc}
\hline $\begin{array}{l}\text { Textura } \\
\text { del suelo }\end{array}$ & $\begin{array}{c}\text { Permeabilidad } \\
\text { saturada } \\
{[\mathrm{cm} / \mathrm{h}]}\end{array}$ & $\begin{array}{c}\text { Total de } \\
\text { espacio poroso } \\
\text { \% por vol. }\end{array}$ & $\begin{array}{c}\text { Capacidad de } \\
\text { campo } \\
\text { \% por vol. }\end{array}$ & $\begin{array}{c}\text { Marchitez } \\
\text { permanente } \\
\text { \% por vol. }\end{array}$ \\
\hline Arenoso & 5 & 38 & 15 & 7 \\
& $(2,5-25,5)$ & $(32-42)$ & $(9-22)$ & $(3-11)$ \\
Franco-arenoso & 2.5 & 43 & 21 & 9 \\
& $(1,3-7,6)$ & $(40-47)$ & $(14-29)$ & $(6-13)$ \\
Franco & 1,3 & 47 & 31 & 14 \\
& $(0,8-2,0)$ & $(43-49)$ & $(24-39)$ & $(11-18)$ \\
Franco arcilloso & 0,8 & 49 & 36 & 18 \\
& $(0,25-1,5)$ & $(47-41)$ & $(30-43)$ & 20 \\
Arcillo-arenoso & 0,25 & 51 & 40 & $(16-23)$ \\
& $(0,03-0,5)$ & $(49-53)$ & $(34-47)$ & 21 \\
Arcilloso & 0,05 & 53 & 44 & $(17-26)$ \\
\hline
\end{tabular}

\section{CONFIABILIDAD DEL MODELO}

Para deteterminar la confiabilidad del modelo ZONOSA, se comparó, con los resultado obtenidos con el modelo MARTHE (BRGM-INSIVUMEH, 1997). Además dicho proyecto realizó en el campo medidas de humedad a diferentes profundidades. Los parámetros de ingreso en el modelo ZONOSA, son los mismos que fueron utilizados en el modelo MARTHE. Los resultados obtenidos se muestran en la figura 2

\section{CONCLUSIONES}

Comparando el modelo ZONOSA con el MARTHE y los resultados de campo obtenidos, se deduce que para las condiciones del suelo en el sitio en que realizó el estudio BRGM-INSIVUMEH (1997), el modelo ZONOSA se ajustó mejor a los datos de campo a las profundidades de $15 \mathrm{~cm}$ y $130 \mathrm{~cm}$. El modelo MARTHE, se ajustó mejor a la profundidad de $70 \mathrm{~cm}$. Esta diferencia se debe posiblemente por considerar el ZONOSA un medio isotrópico. De los resultados anteriores se puede considerar el modelo ZONOSA una buena herramienta para el estudio de las aguas subterráneas en la zona no saturada. Este modelo no considera las variaciones de permeabilidad vertical en las diferentes celdas de cada capa.

\section{Limitaciones del modelo}

El modelo esta limitado a dos capas y medios isotrópicos, además cada capa es considerada homogénea. Otra limitación se debe a que el modelo es unidimensional, sin considerar el flujo horizontal en el balance de humedades. Sin embargo, la recarga de los acuíferos se debe principamente a la lluvia y no a una recarga puntual, por lo que se puede considerar que el flujo en la zona no saturada es vertical, a menos que exista flujo subsuperficial, que produzca flujos horizontales en una celda dada. 


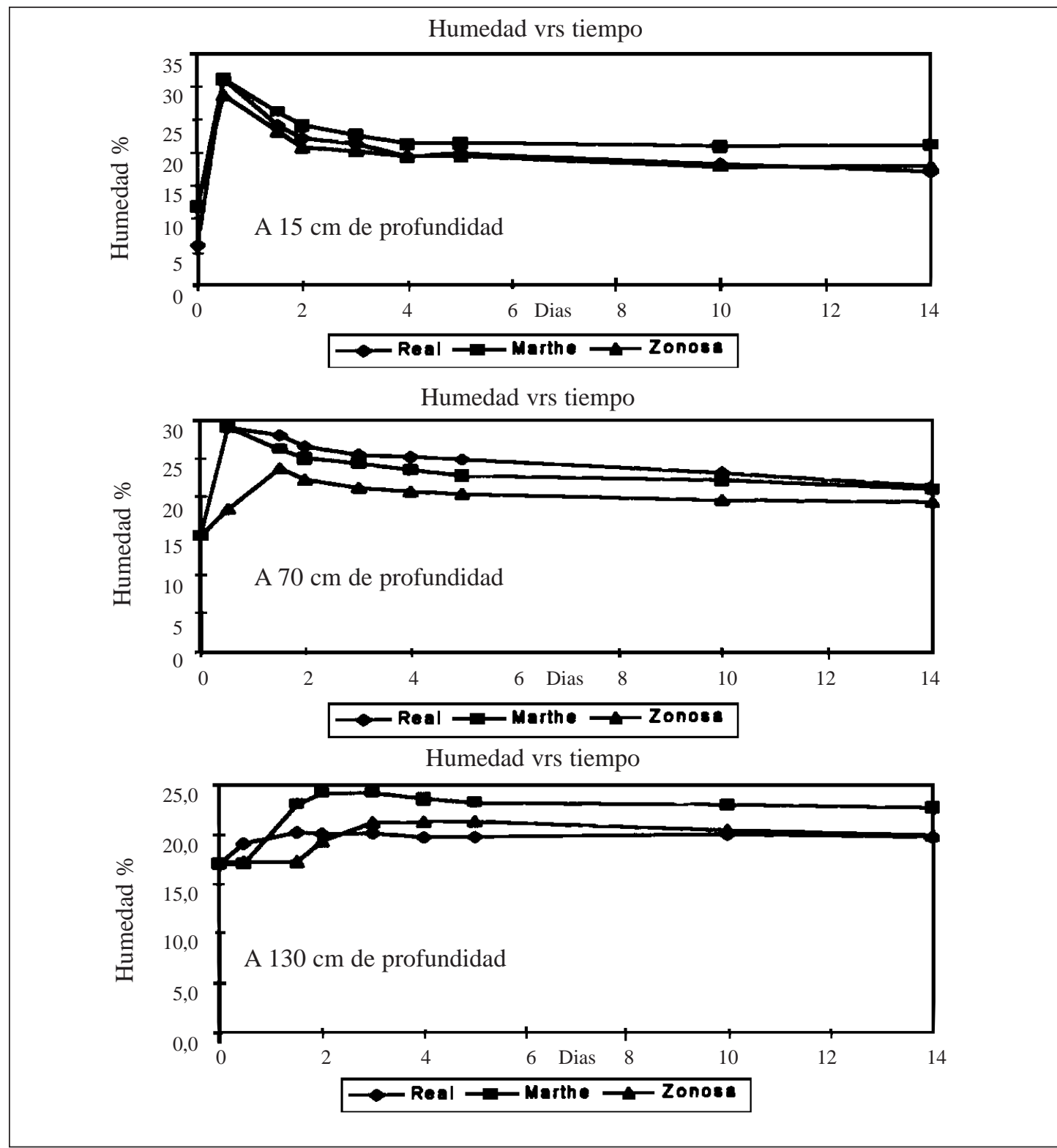

Fig. 2: Comparación de los resultados de humedad obtenidos con el modelo ZONOSA, MARTHE y datos de campo, a diferentes profundidades.

\section{AGRADECIMIENTOS}

Agradezco el apoyo y colaboración recibida del MSc. Marcelino Losilla, por los acertados consejos y observaciones para desarrollar y presentar el modelo en forma amigable y de uso práctico. Además, no quiero dejar de agradecer la constante revisión al mismo, durante la etapa de elaboración. 


\section{REFERENCIAS}

CANDELA, L. \& VARELA, M., 1993: La zona no saturada y la contaminación de las aguas subterráneas.- 322 págs., CIMNE, Barcelona.

FETTER, C.W., 1994: Applied Hydrogeology.691 págs, Prentice Hall, Englewood Cliffs.

FREEZE, R.A. \& CHERRY, J.A., 1979:

Groundwater.- 604 págs, Prentice Hall, Englewood Cleefs.
JEGAT, A., 1979: Aprovechamiento y modelos de aguas subterráneas.

BRGM-INSIVUMEH, 1997: Modelización del transporte de agua y solutos en los estratos volcánicos y sedimentarios en la Ciudad de Guatemala.- 144 págs, Proyecto de Cooperación Internacional Franco-Guatemalteco, Guatemala. 